\title{
Autoeficácia de idosos iniciantes em um programa de exercícios físicos: comparação entre permanecentes e não permanecentes
}

Self-efficacy of elderly beginners in a physical exercise program: comparison between persevering and not persevering

Simone Teresinha Meurer ${ }^{1}$

Lucélia Justino Borges ${ }^{1}$

Susane Graup ${ }^{2}$

Tânia Rosane Bertoldo Benedetti ${ }^{1,3}$

\section{RESUMO}

Objetivou-se analisar a autoeficácia (AE) para a prática de atividade física de idosos iniciantes em um programa de exercícios físicos (PEF). O PEF foi oferecido no contexto comunitário de Florianópolis - SC, durante três meses (três vezes/semana; 60 min/sessão) e foi conduzido por profissionais de Educação Física vinculados à rede de Atenção Primária à Saúde. Participaram do estudo 51 idosos (41 mulheres). A coleta de dados foi realizada por meio de questionários e envolveu variáveis sociodemográficas, percepção do estado de saúde e autoeficácia. Foram considerados "permanecentes" todos os idosos que participaram do PEF até o seu término e, "não permanecentes" aqueles que em algum momento deixaram de participar. Teste Qui-Quadrado ou Exato de Fisher foram utilizados para a comparação dos resultados, adotando nível de significância de 5\%. Os principais resultados revelam que há diferenças da $\mathrm{AE}$ para a caminhada entre idosos permanecentes e não permanecentes do PEF ( $\mathrm{p}=0,02)$, sendo superior entre os permanecentes. Além disso, a maioria dos avaliados percebe que o estado de saúde não dificulta a prática de $\mathrm{AF}$. Os resultados obtidos sugerem que a $\mathrm{AE}$ contribuiu para a permanência de idosos no PEF e, dessa forma, sua avaliação no início de intervenções torna-se uma ferramenta importante no sentindo de desenvolver estratégias que auxiliem os participantes a incrementarem a percepção da $\mathrm{AE}$.

\section{PALAVRAS-CHAVE}

Autoeficácia; Adoção; Manutenção; Idosos.

\begin{abstract}
The aim of this study was to analyze the self-efficacy (AE) for physical activity among elderly beginners in a physical exercise program (PEP). The PEP was offered in the community of Florianópolis - SC, during three months (three sessions per week, 60 min per session) and was conducted by physical education professionals affiliated with the Primary Care Network. The study included 51 elderly (41 women). Data collection was conducted through questionnaires and involved sociodemographic variables, perceived health status and AE. All elderly who participated in the PEP to its end were considered "persevering"; those who at some point stopped were considered "not persevering". Chi-square or Fisher's exact test were used to compare the results, adopting a significance level of $5 \%$. The main results reveal that are differences of $A E$ for the walk between "persevering" and "not persevering" elderly on $\operatorname{PEP}(p=0.02)$, being higher among "persevering". Additionally, most of the participants evaluated perceive that the practice of AF is not prevented by state of health. The results suggest that SE contributed to the participants permanence in PEP and thus its evaluation in early intervention becomes an important tool in developing strategies that aid participants to increase the perception of $A E$.
\end{abstract}

\section{KEYWORDS}

Self-efficacy; Adoption; Maintenance; Elderly.
Rev Bras Ativ Fís Saúde p. 57-64 DOI

http://dx.doi.org/10.12820/rbafs.v.20n1p57

1 Universidade Federal de Santa Catarina. Núcleo de Cineantropometria e Desempenho Humano e Núcleo de Atividade Física e Saúde. Florianópolis, Santa Catarina, Brasil.

2 Universidade Federal do Pampa/ Campus Uruguaiana. Uruguaiana, Rio Grande do Sul, Brasil.

3 Universidade Federal de Santa Catarina. Departamento de Educação Física. Florianópolis, Santa Catarina, Brasil. 


\section{INTRODUCCÃO}

A autoeficácia (AE) refere-se à confiança ou crença do indivíduo para a realização de determinada atividade com sucesso ${ }^{1}$ e tem demonstrado ser um importante preditor de diferentes comportamentos de saúde ${ }^{2}$, dentre eles, a prática de atividade física $(\mathrm{AF})^{3,4}$.

$\mathrm{A}$ aderência à $\mathrm{AF}$ é um processo dinâmico e complexo, influenciado por diferentes dimensões ${ }^{5}$, sendo a $\mathrm{AE}$ apontada como um dos fatores essenciais para a adoção e a manutenção em programas ${ }^{3}$. A percepção da $\mathrm{AE}$ pode influenciar as atividades realizadas, o tempo investido e a persistência em manter as mesmas, sobretudo diante das dificuldades para a manutenção do comportamento ativo ${ }^{6}$.

Dentre as dificuldades para a adesão e manutenção de idosos à programas estruturados de exercício físico no decorrer do envelhecimento, fase da vida que, muitas vezes, é marcado pela presença de doenças ${ }^{7-9}$, estão a autoavaliação negativa da saúde e a percepção negativa do efeito do exercício físico, em especial, pelo relato de dor e cansaço ${ }^{10}$. No entanto, de acordo com Rabelo e Cardoso $^{11}$, a percepção da saúde e o ajustamento à dor são influenciados pela percepção da $\mathrm{AE}$, sendo que quanto maior a percepção de $\mathrm{AE}$, melhor é a saúde percebida e o ajustamento à dor, bem como maior o esforço despendido para a realização de atividades.

Assim, considerando a importância da $\mathrm{AE}$ sobre a percepção do estado de saúde e adoção de comportamentos ativos na população idosa, torna-se interessante, no contexto da promoção da AF, o entendimento de como os participantes diferem dos não participantes ${ }^{12}$, explorando, principalmente, as diferenças da $\mathrm{AE}$ entre estes. Destaca-se que a avaliação da $\mathrm{AE}$ no início das intervenções com exercício físico, por exemplo, podem contribuir para o desenvolvimento de estratégias que poderão elevar a $\mathrm{AE}$ dos participantes, auxiliando no processo de manutenção desses e, por conseguinte, aumentar a efetividade dessas intervenções ${ }^{13}$. Diante disso, espera-se que o presente estudo possa contribuir para a produção de discussões e conhecimentos relacionados à $\mathrm{AE}$, pela sua originalidade ao envolver programa desenvolvido em contexto comunitário da Atenção Básica e analisar tanto aqueles que permaneceram quanto os que não permaneceram no programa.

Diante disso, objetivou-se analisar a AE de idosos iniciantes em um Programa de Exercícios Físicos (PEF) realizado no contexto comunitário de uma capital do sul do Brasil.

\section{MÉTODOS}

O presente estudo utiliza dados da avaliação baseline do projeto atualmente denominado VAMOS - "Vida Ativa Melhorando a Saúde", que é uma pesquisa colaborativa entre Brasil (Centro de Desportos/Universidade Federal de Santa Catarina) e Estados Unidos (University of Illinois at Urbana-Champaign).

O referido projeto tem como objetivo principal testar a implantação de um programa de promoção da AF por meio de mudança de comportamento e, para tal, aleatoriamente, foram identificadas duas Unidades Básicas de Saúde (UBS) que ofereceram o programa de mudança de comportamento, duas que ofereceram o PEF e duas para grupo controle. A população alvo do estudo 
foram os idosos usuários dessas UBS nos últimos seis meses. Para definição da amostra foram adotados os seguintes critérios de inclusão: ter 60 anos de idade ou mais, não apresentar debilidade física e/ou mental severa, não ter participado de grupos de atividades físicas nos últimos seis meses e apresentar interesse em participar da pesquisa. Maior detalhamento metodológico foi descrito por Benedetti e col. ${ }^{14}$.

O PEF foi oferecido durante três meses, três vezes por semana, com duração de $60 \mathrm{~min} / \mathrm{sessão,} \mathrm{totalizando} 36$ sessões, com o objetivo de desenvolver componentes da aptidão física relacionada à saúde. As aulas dividiam-se em três partes: inicial (aquecimento); principal (desenvolvimento das capacidades físicas) e final (alongamento) e consistiam, principalmente, em atividades de ginástica com intensidade leve à moderada, com uso de materiais como bastões, halteres, caneleiras, bolas, colchonetes e arcos. Profissionais de Educação Física vinculados à rede de Atenção Primária à Saúde do município de Florianópolis foram responsáveis pela condução das aulas.

Para esta investigação, a amostra foi composta por todos os idosos que iniciaram a participação no PEF e que realizaram as avaliações de baseline. Assim, participaram 51 idosos (41 mulheres), com idade entre 60 e 88 anos.

A coleta de dados, conduzida nos meses de junho e julho de 2012 por profissionais e acadêmicos de Educação Física previamente treinados, foi realizada por meio de questionários aplicados em forma de entrevista. As informações sociodemográficas (sexo, escolaridade, idade, estado civil) foram relatadas pelos idosos durante a entrevista.

A percepção dos participantes sobre o estado de saúde e a dificuldade ou não para a prática de AF foi obtida a partir da pergunta - "Para o(a) $\operatorname{sr}(\mathrm{a})$, seu estado de saúde atual dificulta a prática de atividade física?”. As opções de respostas eram "sim" ou "não".

A AE foi avaliada por meio de uma escala de autoeficácia para atividade física, validada para idosos ${ }^{15}$, sendo que a escala original apresentou validade e consistência interna em adultos brasileiros demonstrando características psicométricas adequadas ${ }^{16}$. Essa escala avalia separadamente a AE para a caminhada e para atividades físicas moderadas e vigorosas (AFMV) e é composta por quatro itens com repostas dicotômicas ("não" versus "sim"). Assim, o escore geral da escala varia de "zero" a "quatro" pontos. O estudo de validação da escala para idosos ${ }^{15}$ não sugere pontos de corte, mas indica que quanto maior o escore, maior a $\mathrm{AE}$. No presente estudo adotou-se a média dos escores como ponto de corte para categorizar os dados. Assim, escores de AE inferiores a dois foram classificados como "baixa" AE e, escores superiores, como "elevada" AE.

Foram considerados "permanecentes" todos os idosos que participaram do PEF até o seu término e, "não permanecentes" aqueles que, independente do motivo, deixaram de participar do PEF em algum momento. Destaca-se que não foram investigados os motivos da "não permanência" e que a frequência nas aulas do PEF não foram consideradas para a classificação de permanecentes.

A análise dos dados foi realizada de forma descritiva (frequência relativa e absoluta) e, para a comparação das categorias de AE entre os grupos (permanecentes e não-permanecentes; dificulta a prática de AF e não dificulta) utilizou-se o teste Qui-Quadrado ou Exato de Fisher. As análises foram realizadas no software SPSS, versão $15.0^{\circledR}$ e foi adotado nível de significância de $5 \%$. 
A pesquisa atendeu preceitos éticos e foi aprovado na Comissão Nacional de Ética em Pesquisa ( $n^{\circ}$ 480.560) e no Comitê de Ética em Pesquisa com Seres Humanos, da Universidade Federal de Santa Catarina, sob o parecer n 2387.

\section{RESULTADOS}

Participaram do presente estudo 51 idosos, desses, 30 permaneceram no PEF durante o período de estudo.

Na tabela 1, são apresentadas as características sociodemográficas, a percepção sobre a influência do estado de saúde atual sobre a prática de AF, bem com, a comparação dessas variáveis entre os permanecentes e não permanecentes no PEF.

Identificou-se que a maioria dos participantes tem baixa escolaridade, são casados ou em união estável e percebem que o estado de saúde não dificulta a prática de AF. Além disso, observou-se que não existe diferença estatisticamente significante nas variáveis sociodemográficas e na percepção que o estado de saúde dificulta a prática de $\mathrm{AF}$ entre os idosos permanecentes e não permanecentes no PEF.

TABELA 1 - Comparação das características sociodemográficas e percepção sobre a influência do estado de saúde sobre a prática de AF entre idosos permanecentes e não permanecentes em um PEF.

\begin{tabular}{lcccc}
\hline & Total & Permanecentes & Não Permanecentes & \\
\hline & $n$ & $n(\%)$ & $n(\%)$ & $p\left(\chi^{2}\right)$ \\
\hline Sexo & & & & 0,39 \\
\hline Masculino & 10 & $05(16,7)$ & $05(23,8)$ & \\
\hline Feminino & 41 & $25(83,3)$ & $16(76,2)$ & \\
\hline Idade lanos) & & & & 0,21 \\
\hline 60 - 69 & 24 & $16(53,3)$ & $08(38,0)$ & \\
\hline 70 ou mais & 27 & $14(46,7)$ & $13(62,0)$ & \\
\hline Escolaridade lanos de estudo formal) & & & & \\
\hline Analfabeto (0) & 04 & $02(06,7)$ & $02(09,5)$ & $12(57,1)$ \\
\hline 1 - 8 anos & 35 & $23(76,6)$ & $07(33,3)$ & \\
\hline 9 anos ou mais & 12 & $05(16,7)$ & & 0,36 \\
\hline Estado civil & & & $10(47,6)$ & \\
\hline Com companheiro & 27 & $17(56,6)$ & & \\
\hline Sem companheiro & 24 & $13(34,4)$ & $09(42,9)$ & \\
\hline Estado de Saúde dificulta prática de AF & & & $12(57,1)$ & \\
\hline Sim & 15 & $06(20)$ & & \\
\hline Não & 36 & $24(80)$ & & \\
\hline
\end{tabular}

$\chi^{2}=$ Teste Qui-Quadrado. AF= Atividade Física.

A associação entre a percepção da influência do estado de saúde atual sobre a prática de $\mathrm{AF}$ e a $\mathrm{AE}$ para a $\mathrm{AF}$ está apresentada na Tabela 2.

Os resultados mostram que há associação entre $\mathrm{AE}$ e a percepção da influência do estado de saúde sobre a prática de AF. Dentre os idosos que afirmaram que o estado de saúde não dificulta a prática de $\mathrm{AF}$, a maioria apresentou elevada AE para a caminhada. 
TABELA 2 - Percepção sobre o estado de saúde e a autoeficácia para a AF em idosos.

\begin{tabular}{|c|c|c|c|}
\hline \multirow{3}{*}{ Autoeficácia } & \multicolumn{2}{|c|}{ Estado de Saúde dificulta a AF } & \multirow[b]{3}{*}{$\mathrm{P}$} \\
\hline & Não & Sim & \\
\hline & $\mathrm{n}(\%)$ & $\mathrm{n}(\%)$ & \\
\hline \multicolumn{4}{|l|}{ AE Caminhada } \\
\hline Elevada & $23(76,66)$ & $01(4,2)$ & \multirow{2}{*}{$<0,01 *$} \\
\hline Baixa & $13(43,00)$ & $14(51,9)$ & \\
\hline \multicolumn{4}{|l|}{ AE AFMV } \\
\hline Elevada & $13(81,3)$ & $03(18,8)$ & \multirow{2}{*}{0,21} \\
\hline Baixa & $23(65,7)$ & $12(34,3)$ & \\
\hline
\end{tabular}

* $\chi^{2}(p \leqslant 0,05) . A F=$ Atividade Física; AE= Autoeficácia; AFMV= Atividade Física Moderada e Vigorosa.

A AE para AF, separadamente em AE para caminhada e AFMV está apresentada na Tabela 3 .

Identificou-se diferença estatisticamente significante na percepção da $\mathrm{AE}$ para a caminhada entre permanecentes e não permanecentes do PEF. Dentre os permanecentes a maioria apresentou elevada $\mathrm{AE}$ para a caminhada ao ingressarem no PEF.

TABELA 3 - Autoeficácia para a atividade física em idosos, de acordo com a permanência no PEF.

\begin{tabular}{lccc}
\hline \multirow{2}{*}{ Autoeficácia } & Permanecentes & Não Permanecentes & \\
\cline { 2 - 3 } & $\mathrm{n}(\%)$ & $\mathrm{n}(\%)$ & $\mathrm{p}$ \\
\hline AE Caminhada & & & \\
\hline Elevada & $18(60,0)$ & $06(28,6)$ & $0,02^{*}$ \\
\hline Baixa & $12(40,0)$ & $15(71,4)$ & \\
\hline AE AFMV & & & \\
\hline Elevada & $11(36,7)$ & $05(23,8)$ & 0,25 \\
\hline Baixa & $19(63,3)$ & $16(76,2)$ & \\
\hline
\end{tabular}

* teste $\chi^{2}(p \leqslant 0,05) ; A E=$ Autoeficácia; $A F M V=$ Atividade Física Moderada e Vigorosa.

\section{DISCUSSÃO}

Os resultados do presente estudo mostraram diferenças da AE para a caminhada entre permanecentes e não permanecentes do PEF, bem como elevada AE para os idosos que percebem que o estado de saúde não dificulta a prática de atividades físicas. Vale destacar que 30 idosos permaneceram no PEF durante o período estipulado (três meses) e que os grupos (permanecentes x não permanecentes) não diferiam quanto às características sociodemográficas.

São diferentes as dimensões e fatores que influenciam a permanência e/ou desistência em um $\mathrm{PEF}^{5}$. A literatura aponta que na população idosa um dos principais motivos para a desistência de programas dessa natureza e/ou barreiras para inserção nesses são os problemas relacionados à saúde ${ }^{10,17,18}$.

No presente estudo a maioria dos idosos relatou que o estado de saúde não dificulta a prática de AF e não foram observadas diferenças significantes nessa percepção entre idosos permanecentes e não permanecentes no PEF. No entanto, identificou-se que os idosos com $\mathrm{AE}$ elevada para a caminhada também tiveram percepção mais positiva da saúde ao relatarem que o estado de saúde 
não dificulta a prática de $\mathrm{AF}$, sinalizando relações entre a percepção da saúde e a $\mathrm{AE}$ para a prática de $\mathrm{AF}$.

Tal fato pode ser compreendido considerando a natureza recíproca da $\mathrm{AE}$, que ora se apresenta como determinante e ora como consequência de determinado comportamento ${ }^{20}$. Assim, por um lado, pode-se considerar que AE influencia a percepção do estado de saúde, pois, conforme destacam Rabelo e Cardoso ${ }^{11}$, quanto mais elevada a percepção da $\mathrm{AE}$ melhor é a saúde percebida e o ajustamento à dor. Por outro lado, pode-se considerar que a $\mathrm{AE}$ elevada pode ser decorrente de um melhor estado de saúde, pois, de acordo com estudo realizado com idosos ${ }^{19}$ um melhor estado de saúde físico e mental influenciou a percepção de $\mathrm{AE}$.

Destaca-se ainda que a percepção da $\mathrm{AE}$ não é estática e, portanto, essa pode sofrer modificações ${ }^{2}$, sendo as quatro principais fontes de influência: as experiências diretas, as experiências vicárias, a persuasão social e os estados emocionais e fisiológicos percebidos ${ }^{1,21}$.

Dessa forma, é de suma importância considerar os fatores psicossociais como a AE, bem como as possibilidades para incrementá-la, no desenvolvimento de propostas de intervenções de promoção da saúde ${ }^{22}$, pois, melhorias na percepção da saúde podem contribuir para incrementar a $\mathrm{AE}$, bem como, incrementos da $\mathrm{AE}$ por meio de outras fontes, pode melhorar a percepção da saúde, influenciando diretamente sobre a adesão e manutenção de idosos em programas de educação e promoção em saúde ${ }^{23}$.

Ao analisar a $\mathrm{AE}$ para a AF, foi possível identificar que os idosos permanecentes no $\mathrm{PEF}$ foram aqueles que apresentavam $\mathrm{AE}$ elevada para a caminhada ao iniciarem o programa, sendo significativa a diferença entre os grupos. Tal resultado confirma as proposições da teoria da $\mathrm{AE}^{1}$, sendo que quanto maior a $\mathrm{AE}$, maiores são as possibilidades de adesão e manutenção nessa atividade ${ }^{4,13,22}$. Acredita-se que os idosos com AE mais elevada para a caminhada podem ter tido experiências prévias com essa modalidade, incrementando a $\mathrm{AE}$ para tal atividade bem como a própria aptidão funcional. Além disso, especula-se que ter AE mais elevada pode tê-los deixados mais confiantes para as atividades propostas no PEF, favorecendo a execução das atividades e a motivação de permanecer no programa. Assim, a percepção da $\mathrm{AE}$ pode influenciar a adoção e a manutenção em $\mathrm{PEF}^{3}$ e de comportamento ativo ${ }^{6}$.

Um estudo conduzido com adultos e idosos também demonstrou o papel da AE para a inserção e permanência em um programa de caminhada ${ }^{12}$. Os resultados demonstraram que aquelas pessoas com maiores níveis de $\mathrm{AE}$ para a AF tiveram maiores chances de se matricular, bem como, de completar o programa de caminhada proposto, quando comparados aos seus pares com níveis menores de eficácia para a AF.

Em relação à AFMV, observou-se que, independente de ter permanecido ou não no $\mathrm{PEF}$, maior parte dos idosos teve baixa $\mathrm{AE}$ para esse tipo de atividades ao iniciarem o programa, o que pode explicar a não observância de diferenças entre os grupos. Além disso, acredita-se que a baixa $\mathrm{AE}$ identificada para essas atividades está relacionada ao fato de a prática de atividades em intensidade elevada, especialmente a vigorosa, não ser comum entre idosos ${ }^{24,25}$, podendo representar uma das barreiras para incremento de $\mathrm{AE}$ para tais atividades entre esse público.

A presente investigação identificou que os idosos permanecentes no PEF apresentavam maior $\mathrm{AE}$ para caminhada ao ingressarem quando comparados 
aos não permanecentes. A partir desses resultados, destaca-se a importância da avaliação da $\mathrm{AE}$ em idosos iniciantes numa intervenção de AF. Tal ação poderá contribuir para o planejamento e sistematização de ações objetivando o aumento da $\mathrm{AE}$, favorecendo a permanência desse grupo etário nos programas e a efetividade das intervenções.

Dentre as limitações do estudo, destaca-se o número reduzido da amostra, o que limita o poder dos testes estatísticos utilizados e o uso das análises bivariadas, por não permitir a inclusão de possíveis fatores de confusão, podendo comprometer a validade externa e a interpretação dos resultados. Além disso, a utilização de dados da $\mathrm{AE}$ em um único momento (baseline) pode limitar o entendimento sobre as diferenças entre os grupos investigados e sobre como a participação no $\mathrm{PEF}$ pode ter contribuído para o incremento da AE. Contudo, deve-se destacar como aspectos positivos o contexto comunitário, o lócus (rede de Atenção Primária à Saúde do município); o envolvimento dos profissionais de Educação Física da rede, aproximando a pesquisa da realidade; o processo randomizado para a seleção das Unidades Básicas de Saúde participantes da pesquisa e para a alocação do PEF, o que contribui para a validade externa, bem como o instrumento de avaliação da $\mathrm{AE}$ validado para essa população, avaliando separadamente a AE para a caminhada e para as AFMV.

\section{Agradecimentos}

Ao Instituto Lemann, pelo financiamento concedido à pesquisa colaborativa entre Brasil- Universidade Federal de Santa Catarina e Estados Unidos - University of Illinois at Urbana-Champaign, à Coordenação de Aperfeiçoamento de Pessoal de Nível Superior (CAPES), pela concessão de bolsa de doutorado e ao Conselho Nacional de Desenvolvimento Científico e Tecnológico, pelo financiamente (CNPq. Edital Universal 14/2012. Processo n. 475.075/2012).

\section{Contribuição dos autores}

Meurer ST e Borges LJ auxiliaram no trabalho de campo, na análise e interpretação dos dados e redação do artigo. Graup S auxiliou na análise e interpretação dos dados e na redação do artigo. Benedetti foi responsável pela concepção do projeto VAMOS e pela revisão crítica do conteúdo intelectual do presente artigo. Todos os autores aprovaram a versão final.

\section{REFERÊNCIAS}

1. Bandura A. Self-Efficacy: The Exercise of Control. Freeman, New York, 1997.

2. Ashford S, Edmunds J, French DP. What is the best way to change self-efficacy to promote lifestyle and recreational physical activity? A systematic review with metaanalysis. Br J Health Psychol. 2010;15(2):265-88.

3. McAuley E, Jerome GJ, Elavsky S, Marquez DX, Ramsey SN. Predicting long-term maintenance of physical activity in older adults. Prev Med. 2003; 37(2):110-8.

4. Wójcicki TR, McAuley E. Manutenção de Intervenções de Atividade Física: premissas para eficácia individual. Rev Família, Ciclos Vida e Saúde no Context Soc. 2014;2(2):182-7.

5. Barros MB, Iaochite RT. Autoeficácia para a prática de atividade física por indivíduos adultos. Rev Motri. 2012;8(2):32-41.

6. Becofsky K, Baruth M, Wilcox S. Psychosocial Mediators of Two Community-Based Physical Activity Programs. Ann Behav Med. 2014; 48(1):125-9. 
7. Veras R. Prevenção de doenças em idosos: os equívocos dos atuais modelos. Cad. Saúde Pública. 2012; 28(10):1834-40.

8. Kayser B, Miotto C, Dal Molin V, Kummer J, Klein SR, Wibelinge LM. Influência da dor crônica na capacidade funcional do idoso. Rev Dor. 2014;15(1):48-50.

9. Gomes KV, Zazá DC. Motivos de Adesão a Prática de Atividade Física em Idosas. Rev Bras Ativ Fis Saúde. 2009;14(2):132-8.

10. Nascimento MC, Silva OMP da, Saggioratto CML, Vargas KCB, Schopf K, Klunk J. O desafio da adesão aos exercícios físicos em grupos de idosos em Palmitos/SC: Motivos para a prática e para a desistência. Rev Bras Ativ Fis Saúde. 2010;15(3):140-4.

11. Rabelo F, Mendon C. Auto-eficácia , doenças crônicas e incapacidade funcional na velhice. Psico-USF. 2007;12(1):75-81.

12. Jerome GJ, McAuley E. Enrollment and participation in a pilot walking programme: the role of self-efficacy. J Health Psychol. 2013; 18(2):236-44.

13. McAuley E, Mullen SP, Szabo AN, White SM, Wójcicki TR, Mailey EL, et al. Selfregulatory processes and exercise adherence in older adults: executive function and self-efficacy effects. Am J Prev Med. 2011; 41(3):284-90.

14. Benedetti TRB, Schwingel A, Gomez LSR, Chodzko-Zajko W. Programa "VAMOS" (Vida Ativa Melhorando a Saúde): da concepção aos primeiros resultados. Rev. bras. cineantropom. desempenho hum. 2012;14(6):723-37.

15. Borges RA, Rech CR, Meurer ST, Benedetti TRB. Validade e fidedignidade de uma escala para avaliar autoeficácia para a atividade física do idoso. Cad Saúde Pública; no prelo.

16. Rech CR, Sarabia TT, Fermino RC, Hallal PC, Reis RS. Psychometric properties of a self-efficacy scale for physical activity in Brazilian adults. Rev Panam Salud Publica. 2011;29(4):259-66

17. Souza DL de, Vendrúsculo R. Fatores determinantes para a continuidade da participação de idosos em programas de atividade física: a experiência dos participantes do projeto "Sem Fronteiras". Rev Bras Educ Física e Esportes. 2010;24(1):95-105.

18. Cardoso AS, Borges LJ, Mazo GZ, Benedetti TRB, Kuhnen AP. Fatores influentes na desistência de idosos em um programa de exercício físico. Movimento. 2008;14(1):225-39.

19. McAuley E, Konopack JF, Motl RW, Morris KS, Doerksen SE, Rosengren KR. Physical activity and quality of life in older adults: influence of health status and self-efficacy. Ann Behav Med. 2006;31(1):99-103.

20. Rhodes RE, Nigg CR. Advancing physical activity theory: a review and future directions. Exerc Sport Sci Rev. 2011;39(3):113-9.

21. Bandura A. Social foundations of thought and action: a social cognitive theory. Englenwood Cliffs: Prentice Hall; 1986.

22. Lee L-L, Arthur A, Avis M. Using self-efficacy theory to develop interventions that help older people overcome psychological barriers to physical activity: a discussion paper. Int J Nurs Stud. 2008;45(11):1690-9.

23. Silva MCS da, Lautert L. O senso de auto-eficácia na manutençao de comportamentos promotores de saúde de idosos. Rev Esc Enferm da USP. 2010;44(1):61-7.

24. Carvalho ED de, Valadares ALR, Costa-Paiva LH Da, Pedro AO, Morais SS, PintoNeto AM. Atividade física e qualidade de vida em mulheres com 60 anos ou mais: fatores associados. Rev Bras Ginecol e Obs. 2010;32(9):433-40

25. Mazo GZ, Mota J, Gonçalves LHT, Matos M. Nível de atividade física, condiçoes de saúde e caracteríticas sócio-demográficas de mulheres idosas brasileiras. Rev Port Ciência do Desporto. 2005;2(5):202-12.

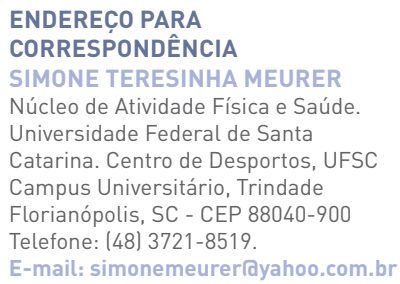

